\title{
Correlation of Ammonia Storage and Dielectric Properties of SCR Catalyst Materials by Microwave Cavity Perturbation
}

\author{
Markus Dietrich ${ }^{1}$, Dieter Rauch ${ }^{1}$, Ulrich Simon ${ }^{2}$, Adrian Porch $^{3}$, Ralf Moos ${ }^{1}$ \\ ${ }^{1}$ Department of Functional Materials, University of Bayreuth, 95440 Bayreuth, Germany \\ Corresponding e-mail address: Functional.Materials@uni-bayreuth.de \\ 2 Institute of Inorganic Chemistry (IAC), RWTH Aachen University, 52074 Aachen, Germany \\ 3 School of Engineering, Cardiff University, Cardiff CF24 3AA, Wales, UK
}

\begin{abstract}
:
The ammonia-based selective catalytic reduction (SCR) has become the major $\mathrm{NO}_{x}$ control strategy for light and heavy diesel engines. Before reducing $\mathrm{NO}_{x}$, storage of ammonia on the active sites of the catalyst is crucial. A better understanding of the ammonia storage behavior is needed for improvements in efficiency and control of the exhaust gas aftertreatment systems. Therefore, the aim of this article is the correlation of the dielectric properties of the SCR material with the amount of stored ammonia. Recently, a laboratory setup using the microwave cavity perturbation method had been developed especially for this purpose and has been significantly improved. This article describes the improvement process and first experimental data. Zeolite-based SCR active material in its $\mathrm{H}$-form was investigated. The powder catalyst sample was monitored by the microwave cavity perturbation method under reaction conditions while storing and depleting ammonia with and without admixed $\mathrm{NO}_{\mathrm{x}}$ at different temperatures. It could be shown that both values of the complex dielectric permittivity are strongly correlated to the stored mass of ammonia. The influence of the temperature on the ammonia storage behavior was described. It could be shown by experiment that the response of the real part and the imaginary part of the complex permittivity to ammonia storage have different temperature dependencies.
\end{abstract}

Key words: microwave cavity perturbation, ammonia SCR, ammonia storage, H-ZSM-5, complex permittivity

\section{Ammonia-based Selective Catalytic Reduction}

In automotive exhaust gas aftertreatment, the ammonia-based SCR uses a non-toxic, aqueous urea solution (AdBlue ${ }^{T M}$ ) as the reducing agent. The injected solution decomposes thermally to ammonia $\left(\mathrm{NH}_{3}\right)$ in the hot exhaust [1]. Before reacting, ammonia adsorbs on the SCR catalyst. This also allows buffering of flow changes and of temperature in order to ensure a permanent $\mathrm{NO}_{x}$ conversion [2]. The catalyst reduces $\mathrm{NO}_{x}$ selectively to nitrogen $\left(\mathrm{N}_{2}\right)$ and water $\left(\mathrm{H}_{2} \mathrm{O}\right)$. The two main SCR reactions are shown in the following: the standard SCR reaction (1) and the fast SCR reaction (2) with equimolar amounts of $\mathrm{NO}$ and $\mathrm{NO}_{2}[3]:$

$$
\begin{aligned}
& 4 \mathrm{NH}_{3}+4 \mathrm{NO}+\mathrm{O}_{2} \rightarrow 4 \mathrm{~N}_{2}+6 \mathrm{H}_{2} \mathrm{O} \\
& 4 \mathrm{NH}_{3}+2 \mathrm{NO}+2 \mathrm{NO}_{2} \rightarrow 4 \mathrm{~N}_{2}+6 \mathrm{H}_{2} \mathrm{O}
\end{aligned}
$$

\section{Microwave Cavity Perturbation}

The microwave cavity perturbation technique uses standing electromagnetic waves (resonances) inside a defined resonator (e.g. a metal can). The presence of a small sample inside the resonator leads to a perturbation of the electromagnetic field distribution. The resulting changes of the resonance frequency ( $\left.f_{\text {res }}\right)$ and quality factor $Q$ are related to the complex dielectric permittivity $\varepsilon=\varepsilon_{1}-\mathrm{j} \varepsilon_{2}$ of the sample: the real part $\varepsilon_{1}$ (or, more correctly the susceptibility, $\left.\varepsilon_{1}-1\right)$ quantifies the polarization of the material and is linked mostly to decreases in the resonance frequency. The imaginary part $\varepsilon_{2}$ quantifies the dielectric losses and is linked to decreases in $Q$. The laboratory setup as described in [4] uses the $\mathrm{TM}_{010}$ cylindrical cavity mode, due to its uniform electric field in the center of the cavity. The insertion of the sample leads to a change of the resonance frequency from $f_{0}$ to $f_{\mathrm{s}}$ and to a change of the unloaded quality factor from $Q_{0}$ to 
Qs. Together with the volume of the sample $V_{s}$ and the effective volume of the resonator $V_{\text {eff }}$ (depending on the analyzed resonance), the complex dielectric permittivity can be calculated by Equations 3 and 4 [5].

$$
\begin{aligned}
& \frac{f_{0}-f_{s}}{f_{0}} \approx\left(\varepsilon_{1}-1\right) \frac{V_{s}}{2 V_{\text {eff }}} \\
& \frac{1}{Q_{s}}-\frac{1}{Q_{0}}=\Delta\left(\frac{1}{Q}\right) \approx \varepsilon_{2} \frac{V_{s}}{V_{\text {eff }}}
\end{aligned}
$$

The mode volume $V_{\text {eff }}$ for the $\mathrm{TM}_{010}$ mode is $26.9 \%$ of the enclosed volume of the cylinder.

\section{Experimental}

The schematic setup is displayed in Figure 1. The process gas is fed through an almost lossless quartz glass tube with a porous frit for powder samples. The second tube, doublewalled and permanently evacuated, feeds a stream of hot air through the gap between the two tubes to heat up the process gas and to minimize thermal conduction to the surrounding resonator. The $\mathrm{TM}_{010}$ mode was excited inductively by means of two loop-terminated coaxial lines around the cavity's perimeter.

Initial heating tests in [4] showed that the resonator heats up by radiation. In spite of the double-walled tube, this causes systematical measurement errors as a result of a positiondependent cavity temperature. Therefore, the resonator has been modified with meandering active water-cooling. The results of the first heating tests without samples and a constant cooling water flow rate are displayed in Figure 2. Figure 2(a) shows recorded transmission spectra of the resonance peaks of the TM010 mode at different process gas temperatures. The resonance frequencies $f_{\text {res }}$ and the unloaded quality factors $Q_{0}$ of the cavity deduced of the resonance peaks are shown in Figure 2(b) and (c). It is obvious that a linear relation between the increasing temperature and the shift of $f_{\text {res }}$ exists, which is in contrast to Qo, which appears to be almost unaffected. $Q$ is inversely proportional to the surface resistance of the metal walls, which itself is proportional to the square root of the electrical resistivity $\rho$. We conclude that the increased resistivity has a negligible effect on $Q$ over the elevated range of cavity temperature seen here. It is shown that the uncontrolled water-cooling is able to minimize the resonator heating, but still a shift of $f_{\text {res }}$ appears resulting of thermal expansion.
Therefore, a controlled water-cooling (or heating) for a constant resonator temperature is an important improvement that will be implemented in the next stage of the experimental development.

The first measurements on zeolite samples shown below were still restricted to constant temperatures up to $300{ }^{\circ} \mathrm{C}$. It is worth noting that because of the unaffected quality factor, temperature programmed desorption experiments up to $300{ }^{\circ} \mathrm{C}$ and the resulting monitoring of $\varepsilon_{2}$ are already possible.

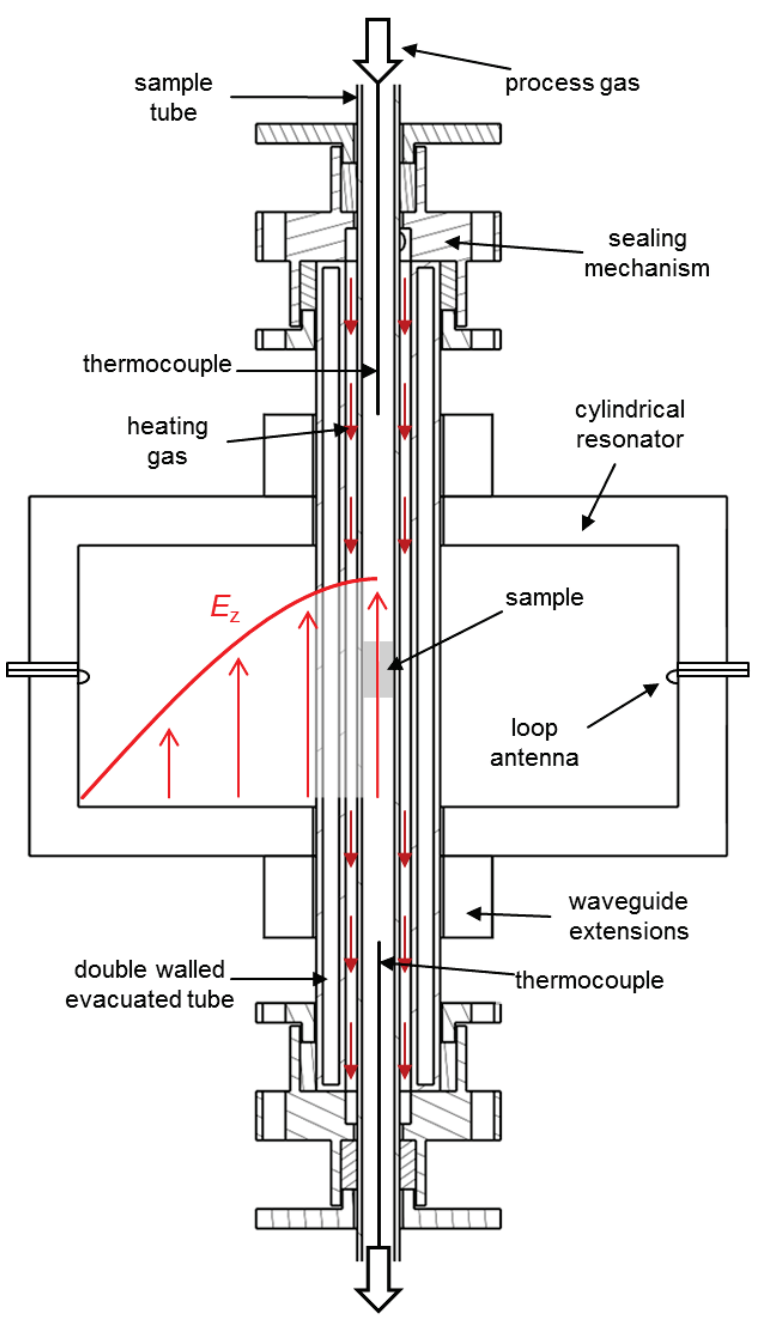

Fig. 1. Schematic setup of the test bench including the sample tube, the double-walled and evacuated tube, the sealing mechanism, the resonator with waveguide extensions, loop antennas and two thermocouples [4]. 

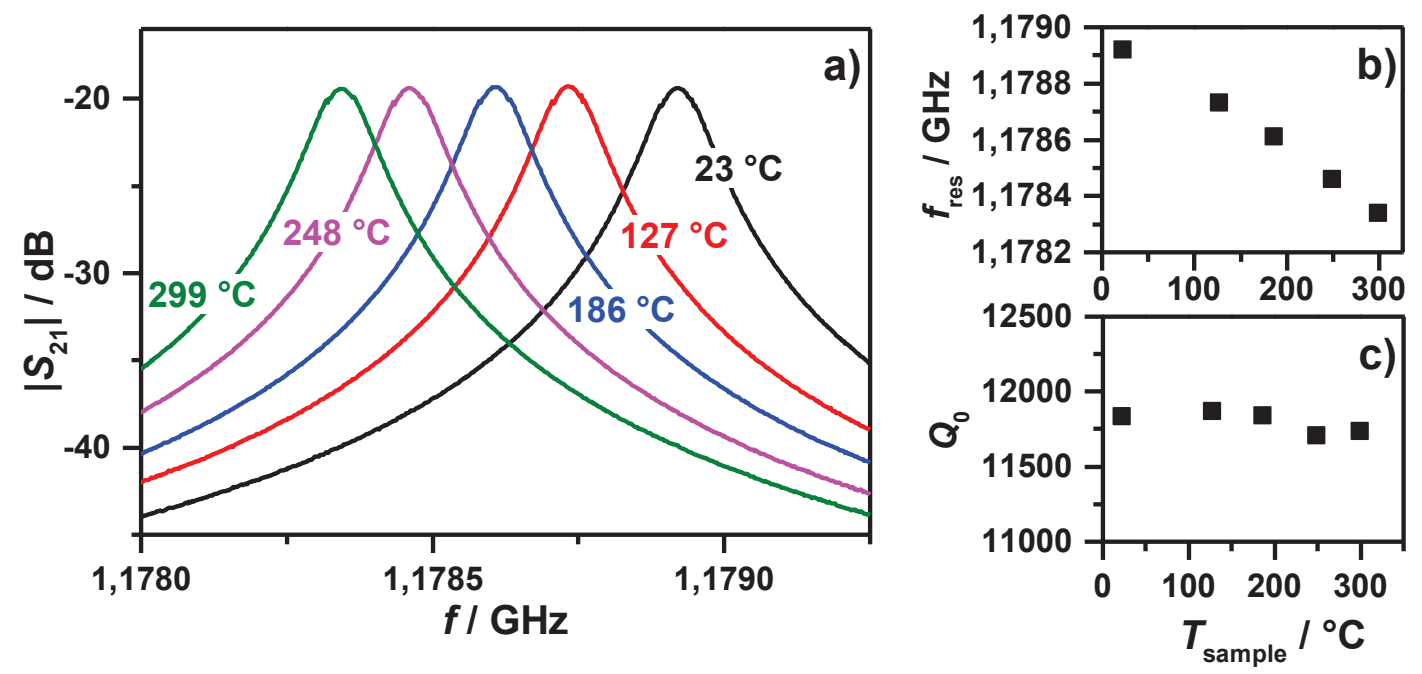

Fig. 2. Temperature effects of the measurement signal without sample with constant cooling water flow: (a) transmission spectra of the TM010 mode at different temperatures at the sample position, (b) resonance frequency and (c) quality factor $(Q)$ as a function of temperature at the sample position.

The sample under investigation was a H-ZSM-5 zeolite with a Si/Al ratio of 27 . The powder was kindly provided by Clariant International Ltd.. The procedure of the ammonia storage experiments for all measurements was identical and is shown for 200,250 , and $300^{\circ} \mathrm{C}$ in the Figures 4(a), (c) and (e). First the samples were heated up to the measurement temperature in nitrogen consisting of $5 \% \mathrm{O}_{2}$ with a total flow of $500 \mathrm{ml} / \mathrm{min}$. Then $500 \mathrm{ppm} \mathrm{NH} \mathrm{N}_{3}$ were admixed. When the downstream $\mathrm{NH}_{3}$ concentration reached the inlet one (determined by FTIR), the $\mathrm{NH}_{3}$ feed was stopped and the weakly bound ammonia could desorb in the base gas. When no more depleting ammonia could be detected downstream of the reactor, equimolar amounts of $175 \mathrm{ppm} \quad \mathrm{NO}$ and $175 \mathrm{ppm} \quad \mathrm{NO}_{2}$ were admixed to the feed gas to deplete the strongly bound ammonia by the fast SCR reaction (2). At the end of the experiment, the samples were again found to be completely free of ammonia.

\section{Results and Discussion}

To show the influence of ammonia loading on the microwave cavity response, three transmission spectra are displayed in Figure 3: the reference spectrum without sample at $250{ }^{\circ} \mathrm{C}$ (black), the spectrum with the ammoniafree sample (red) and the sample loaded with ammonia (blue). Due to the presence of the sample, the resonance frequency shifts, but both peak height (and hence the $Q$ factor) remain constant. This shows that the zeolite sample itself has almost no dielectric losses but has a finite polarization. The spectrum with ammonia loading shows a change in resonance frequency, but also a reduction in peak height (and so a reduction in $Q$ ).

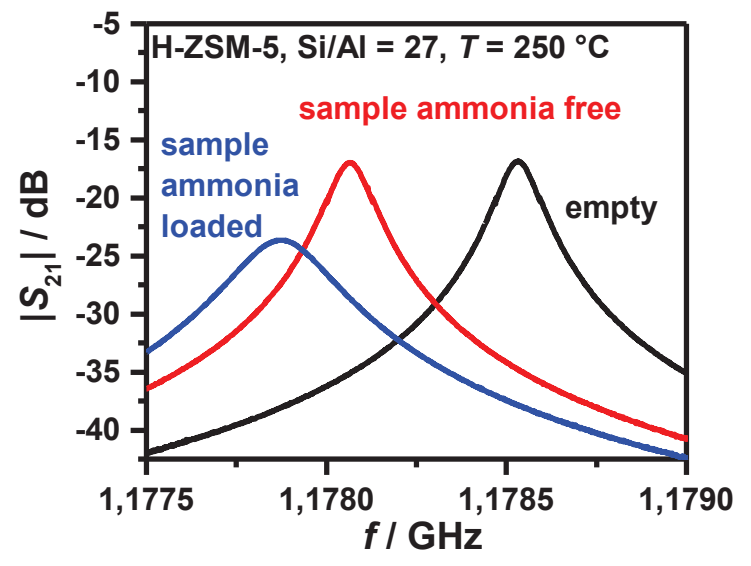

Fig. 3. Transmission spectra of a H-ZSM-5 zeolite, Si/Al ratio of 27 at $250{ }^{\circ} \mathrm{C}$ : empty sample tube as reference (black), sample without ammonia (red) and sample loaded with ammonia (blue).

Figures 4(a), (c), and (e) show the stored amount of $\mathrm{NH}_{3}$ in the sample related to the sample mass, calculated from the difference between the measured upstream and downstream $\mathrm{NH}_{3}$ concentrations, normalized to the sample mass. The ammonia storage capacity decreases with increasing temperature, from $10 \mathrm{mg}$ per gram sample at $200{ }^{\circ} \mathrm{C}$ to $7.5 \mathrm{mg}$ per gram sample at $300^{\circ} \mathrm{C}$.

The results of the permittivity measurements are displayed in Figures 4(b), (d), and (f). It is clearly demonstrated that both $\varepsilon_{1}$ (black) and $\varepsilon_{2}$ (red) are correlated to the mass of stored $\mathrm{NH}_{3}$, but show different responses related to temperature. Without ammonia, $\varepsilon_{1}$ is almost constant at 3.1 for all measured temperatures. The change of $\varepsilon_{1}$ as a result of ammonia storage appears to be almost temperature independent and $\varepsilon_{1}$ changes to 4.0 . $\varepsilon_{2}$ is almost 
zero without ammonia at all temperatures. The response of $\varepsilon_{2}$ to ammonia increases with temperature and its maximum value is 0.45 at $300^{\circ} \mathrm{C}$.

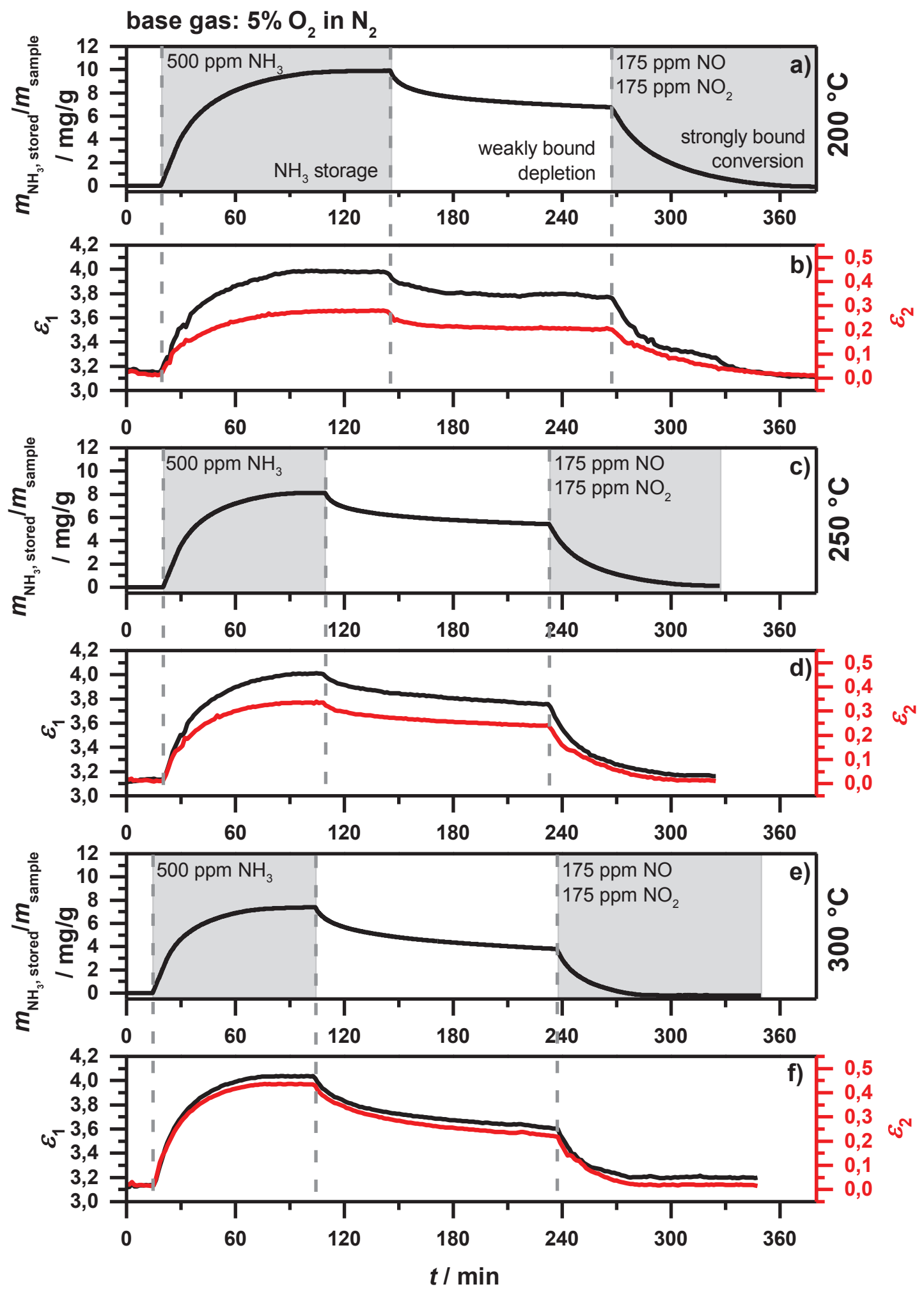

Fig. 4. Measurement for a H-ZSM-5 zeolite with Si/Al=27 at 200, 250 and $300^{\circ} \mathrm{C}:$ (a), (c), and (e) the stored amount of ammonia in the sample, and (b), (d) and (f) the measured complex dielectric permittivity with $\varepsilon_{1}$ (black) and $\varepsilon_{2}(r e d)$. 
$\varepsilon_{1}$ quantifies the polarization, which is mainly determined by the high polarity of the ammonia molecules stored inside the zeolite. This might explain the temperature independence of $\varepsilon_{1}$, as the ammonia content appears to be almost constant in the observed temperature range. The different behavior of $\varepsilon_{2}$, which is a measure for the dielectric loss (which can include conductivity mechanisms for conducting samples) can be explained by an increase of the proton mobility with increasing temperature.

\section{Conclusions and Outlook}

In this work, further developments and first measurements were performed using a recently-introduced set-up [4], on ammonia storage in a $\mathrm{H}-\mathrm{ZSM}-5$ zeolite with a $\mathrm{Si} / \mathrm{Al}$ ratio of 27 in a temperature range from 200 to $300{ }^{\circ} \mathrm{C}$. It could be shown that the ammonia storage can be monitored in both the real and the imaginary part of the complex permittivity. $\varepsilon_{1}$ and $\varepsilon_{2}$ showed different temperature dependences. This offers possibilities for a better understanding of the catalytic and storage site mechanisms of SCR active materials based on zeolites.

In future work we will focus on further improvements to the setup to be able to measure in a higher temperature range and to perform temperature-programmed desorption experiments. Additionally other Si/Al ratios, ionexchanged samples and the influence of the ammonia feed concentration and humidity to the storage behavior will be investigated.

\section{Acknowledgments}

R.M. is indebted to the German Research Foundation (DFG) for supporting this work under grant MO 1060/19-1.

U.S. acknowledges financial supported by the German Research Foundation (DFG), contract No: Si609/14-1, and by the Exploratory Research Space of RWTH Aachen University within the Center for Automotive Catalytic Systems Aachen (ACA).

A.P. acknowledges the support of Merck GKaA.

\section{References}

[1] M. Koebel, M. Elsener, T. Marti, NOx-Reduction in Diesel Exhaust Gas with Urea and Selective Catalytic Reduction, Combust. Sci. and Tech. 121, 85-102 (1996) doi: $10.1080 / 00102209608935588$

[2] G. Busca, L. Lietti, G. Ramis, F. Berti, Chemical and mechanistic aspects of the selective catalytic reduction of $\mathrm{NO}_{x}$ by ammonia over oxide catalysts: A review, Appl. Catal. B: Enviromental 18, 1-36 (1998), doi: 10.1016/S0926-3373(98)00040-X
[3] M. Koebel, M. Elsener, M. Kleemann, Urea-SCR: a promising technique to reduce NOx emissions from automotive diesel engines, Catalysis Today 59, 335-345 (2000), doi: 10.1016/s0920-5861(00)00299-6

[4] M. Dietrich, D. Rauch, A. Porch, R. Moos, A laboratory test setup for in situ measurements of the dielectric properties of catalyst powder samples under reaction conditions by microwave cavity perturbation: set up and initial tests, Sensors 14; 14(9):16856-16868 (2014), doi: $10.3390 / \mathrm{s} 140916856$

[5] A. Porch, D. Slocombe, J. Beutler, P. Edwards, et al., Microwave treatment in oil refining, Appl. Petrochem. Res. 2, 37-44 (2012), doi: $10.1007 / \mathrm{s} 13203-012-0016-4$ 